Research Article

\title{
Optimal Allocation of Combined Renewable Distributed Generation and Capacitor Units for Interconnection Cost Reduction
}

\author{
Saad Ouali $(\mathbb{D}$ and Abdeljebbar Cherkaoui \\ Laboratory of Innovative Technologies (LTI), National School of Applied Sciences, Abdelmalek Esaadi University, \\ Tangier, Morocco \\ Correspondence should be addressed to Saad Ouali; saad.ouali1@gmail.com
}

Received 30 March 2020; Revised 13 June 2020; Accepted 23 June 2020; Published 23 July 2020

Academic Editor: François Vallée

Copyright ( $) 2020$ Saad Ouali and Abdeljebbar Cherkaoui. This is an open access article distributed under the Creative Commons Attribution License, which permits unrestricted use, distribution, and reproduction in any medium, provided the original work is properly cited.

\begin{abstract}
In this paper, a new methodology for the optimal investment in distributed generation is presented, based on an optimal allocation of combined DG and capacitor units to alleviate network voltage constraints and reduce the interconnection cost of renewable generation integration in public medium voltage distribution networks. An analytical optimization method is developed, with the inclusion of practical considerations that are typically neglected in developed works: network topology reconfiguration and the geographical data of the generation land-use and network infrastructure. Powerful results concluded from a sensitivity analysis study of the most impacted parts of the network by the variation of active and reactive power injection under network topology reconfiguration are used as a basis for capacitor units placement. A case study, with two meshed IEEE 15-bus feeders and a new DG to connect, geographical dispersed, are used to simulate the performance of the proposed approach. A cost evaluation of the obtained results proves the effectiveness of the proposed approach to reduce the required charges for connecting new renewable generation units in medium voltage distribution system.
\end{abstract}

\section{Introduction}

Several optimization efforts had been presented in literature with a main aim of maximizing the benefits expected from connecting DGs to electrical networks, by optimizing their location with a defined specific capacity [1], optimizing their size with a defined location [2], optimization of combined location and size [3], and also the identification of the optimal type of DGs to connect [4]. To improve a particular objective or a combination of objectives, the principal searched benefits in literature are minimization of power losses [5], enhancement in voltage profile [6], voltage stability improvement [7], cost minimization [8], maximization of profit [9], reliability enhancement [10], social welfare maximization [11], system average interruption duration index SAIDI improvement [12], and the maximization of the distributed generation capacity [13].
To solve their optimization problem, authors had developed several methods and approaches, which can be classified in four principal categories: analysis approaches [14], mathematical programming algorithms (such as sequential quadratic programming [15], linear programming [16], and nonlinear programming [17]), heuristic methods (such as Particle Swarm Optimization [18], Cuckoo Search Algorithm [19], Genetic Algorithm [20], Karman Filter algorithm [21], Differential Evolution [22], Chaotic Symbolic Organisms Search [23], Tabu Search [24], Harmony Search [25], Firefly algorithm [26], Simulated Annealing [27], Grey wolf optimizer [28], Modified Shuffled Leaping Algorithm [29], Big Bang Big Crunch algorithm [30], Bacterial Foraging Optimization algorithm [31], Ant Colony Optimization [32], Hereford Ranch algorithm [33], Artificial bee colony [34], and Modified Honey Bee Mating algorithm [35]). And hybrid algorithms with a combination of different 
methodologies (such as Genetic Algorithm and Particle Swarm Optimization [36], Genetic Algorithm and Tabu Search [37], and Genetic and Simulated Annealing algorithms [38]).

In this paper, a novel objective function is introduced in the research field area of the integration of distributed generation in power systems, with the development of a powerful optimization method for identifying the optimal combined solution of underground cables and/or overhead lines to lay and capacitor units to install, for reducing the interconnection charges of the generation unit.

Several investment models were presented in literature, investment costs, substation expansion investment cost, operation and maintenance costs, energy losses cost, and the cost of the power purchased from the transmission system. In [39], a stochastic investment model for renewable generation in distribution has been developed to deal with uncertainties in distribution system expansion planning. In [40], the power losses costs have been estimated to devote the optimal operation of MV distribution networks with the presence of DGs units. In this paper, a further novel investment model is introduced, the interconnection of a renewable distributed generation, which represents the cost required for the construction of overhead lines and underground cables between the generation land-use and connection point in the distribution network.

The proposed interconnection cost reduction methodology is based on the assumption that the cost of capacitor bank is lower compared to the costs required for underground MV cables lying and overhead MV lines development. The optimal allocation of the combined renewable generation and capacitor banks is based on powerful observations concluded from a sensitivity analysis study in Section 3 of the most impacted part of the network by the variation of active and reactive power variations, under network topology reconfigurations. Several local and decentralized voltage control methods are developed in literature and can be used to regulate the reactive power of the additional capacity banks with the variation of the produced power of the DG [41, 42].

The main contributions of this paper are as follows:

(i) Introduction of a novel objective to the research field area of optimal allocation of distributed generation

(ii) Inclusion of practical considerations that are typically neglected in already developed works

(iii) Incorporating constraints related to geographical generation land use

(iv) Report results that have the potential to incite researches to develop efficient efforts and encourage private investors to build renewable distributed generation by minimizing the costs of interconnecting their generation units in public networks

(v) The presentation of a powerful tool that could be used directly by distribution electric utilities for the orientation study, as tool able to offer more options to connect a new DG to their electrical networks

\section{Distributed Generation and Public Medium Voltage Distribution Networks}

Several types of distributed generation can be distinguished, according to the technology, type of generators used, and depending on which primary energy they are exploiting; generally 2 principal types of distributed generation can be differentiating: conventional or traditional generators based on combustion engines, and nonconventional generator as follows: storage devices, electrochemical devices, and renewable device.

For connecting a new DG to a medium voltage public distribution network, as the case in Morocco, and according to the law $58-15$, opening access to low and medium voltage networks and the decree No. 2-15-772 on access to the national grid of medium voltage of Morocco, the DG owner will have to address a formal request to the distribution utility.

Distribution utility conducts an orientation study to verify the feasibility of the integration of the new DG, by identifying and evaluating all possible options.

However, the efforts presented in literature, for optimally placing distributed generation, usually neglect the fact that DGs are developed in large surface area, and the choice of the DG site is influenced by several economic, climatic, and geographical conditions. Also, no work had considered the geographical data of the network infrastructure and the DG site.

To better model the problem and to develop an approach adequate for an implementation in public medium voltage distribution networks, renewable distributed generation unit is divided into three different items:

(i) DG site: the land area where the DG is developed

(ii) Connection point: a point from the network cables and lines on where the DG will be connected

(iii) Electrical connection: the overhead lines or underground cables to develop for linking the DG site to the connection point

In the case study presented in Figure 1, seven options are presented to connect the new DGs to the network, with seven connection points and seven electrical connections.

In Figure 1, "CBi" represents the possible connection points, and dotted lines represent the electrical connections to develop for connecting the DG unit.

\section{Optimal Allocation of Capacitor Units and Network Topology Reconfigurations}

Another shortcoming in the efforts [1-42] is the network topology reconfigurations. Medium voltage distribution systems are usually designed as mesh networks but operate in a radial structure. The network topology of distribution systems can be changed for maintenance activities, emergency operations, or network 


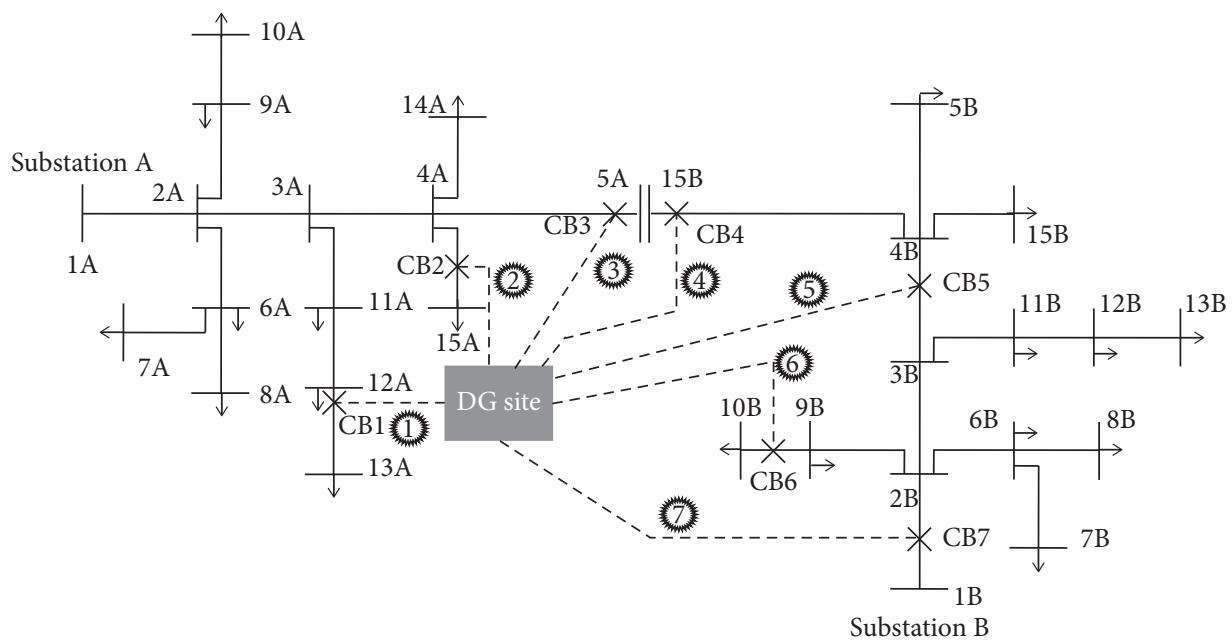

FIGURE 1: Single line diagram of the case study (topology structure no. 1).

reconfiguration, in some cases, for several times in a same day. The optimal access point in a defined structure may be the point to avoid in other structures. The connection point of DGs, loads, capacitor banks, or any other electrical equipment should be done considering network topology reconfigurations, and all the scenario of the network exploration.

However, powerful observations could be concluded from an analysis study of the most impacted part of the network by the new DG with network topology reconfigurations.

Before connecting the DG unit to the distribution network, the relation between the variation in network voltage and the variation in active and reactive of the 15 buses:

$$
\left[\begin{array}{c}
\mathrm{d} V_{1 \mathrm{~A}} \\
\ldots \\
\mathrm{d} V_{15 \mathrm{~A}}
\end{array}\right]=\left[\begin{array}{cccccc}
\frac{\partial V_{1}}{\partial P_{1}} & \ldots & \frac{\partial V_{n}}{\partial P_{1}} & \frac{\partial V_{1}}{\partial Q_{1}} & \ldots & \frac{\partial V_{n}}{\partial Q_{1}} \\
\ldots & \ldots & \ldots & \ldots & \ldots & \ldots \\
\frac{\partial V_{1}}{\partial P_{n}} & \ldots & \frac{\partial V_{n}}{\partial P_{n}} & \frac{\partial V_{1}}{\partial Q_{n}} & \ldots & \frac{\partial V_{n}}{\partial Q_{n}}
\end{array}\right] \times\left[\begin{array}{c}
\mathrm{d} P_{1 \mathrm{~A}} \\
\ldots \\
\mathrm{d} P_{15 \mathrm{~A}} \\
\mathrm{~d} Q_{1 \mathrm{~A}} \\
\ldots \\
\mathrm{d} Q_{15 \mathrm{~A}}
\end{array}\right] .
$$

After the connection of the DG at the CB1, the IEEE 15bus network becomes a feeder with 16 buses and the relation between the total derivation of network voltage and the variation of active and reactive become $\left[\begin{array}{c}\mathrm{d} V_{1 \mathrm{~A}} \\ \cdots \\ \mathrm{d} V_{\mathrm{DG}} \\ \cdots \\ \mathrm{d} V_{15 \mathrm{~A}}\end{array}\right]=\left[\begin{array}{cccccc}\frac{\partial V_{1}}{\partial P_{1}} & \cdots & \frac{\partial V_{n}}{\partial P_{1}} & \frac{\partial V_{1}}{\partial \mathrm{Q}_{1}} & \cdots & \frac{\partial V_{n}}{\partial \mathrm{Q}_{1}} \\ \cdots & \cdots & \cdots & \cdots & \cdots & \cdots \\ \frac{\partial V_{1}}{\partial P_{\mathrm{DG}}} & \cdots & \frac{\partial V_{n}}{\partial P_{\mathrm{DG}}} & \frac{\partial V_{1}}{\partial \mathrm{Q}_{\mathrm{DG}}} & \cdots & \frac{\partial V_{n}}{\partial \mathrm{Q}_{\mathrm{DG}}} \\ \cdots & \cdots & \cdots & \cdots & \cdots & \cdots \\ \frac{\partial V_{1}}{\partial P_{n}} & \cdots & \frac{\partial V_{n}}{\partial P_{n}} & \frac{\partial V_{1}}{\partial Q_{n}} & \cdots & \frac{\partial V_{n}}{\partial Q_{n}}\end{array}\right] \times\left[\begin{array}{c}\mathrm{d} P_{1} \\ \cdots \\ \mathrm{d} P_{\mathrm{DG}} \\ \cdots \\ \mathrm{d} P_{n} \\ \mathrm{~d} Q_{1} \\ \cdots \\ \mathrm{d} Q_{\mathrm{DG}} \\ \cdots \\ \mathrm{d} Q_{n}\end{array}\right]$.

In Figures 1-3, three different topologies structures are presented resulting of the case study; in Figure 1, the switch at the node " $5 \mathrm{~A}-15 \mathrm{~B}$ " is opened and the topology structure of Figure 2 is obtained by closing the opened switch at node " $5 \mathrm{~A}-15 \mathrm{~B}$ " and opening the sectionalize switch at the node "3B," and in Figure 3, the opened is moving to the node "2A." The DG is supposed to be connected at CB1.

Let's now evaluate the most impacted parts of the networks by the variation of the injection active power and the variation of injected or consumed reactive of the DG. The results obtained are arranged in a descending order, and the most 7 impacted nodes are reported in Table 1.

Table 1 shows that the impact of a new DG on network voltage changes with network topology structure reconfiguration, and the influence of connecting a DG in a defined connection point may increase with network topology reconfigurations, as the example of connecting the DG at 


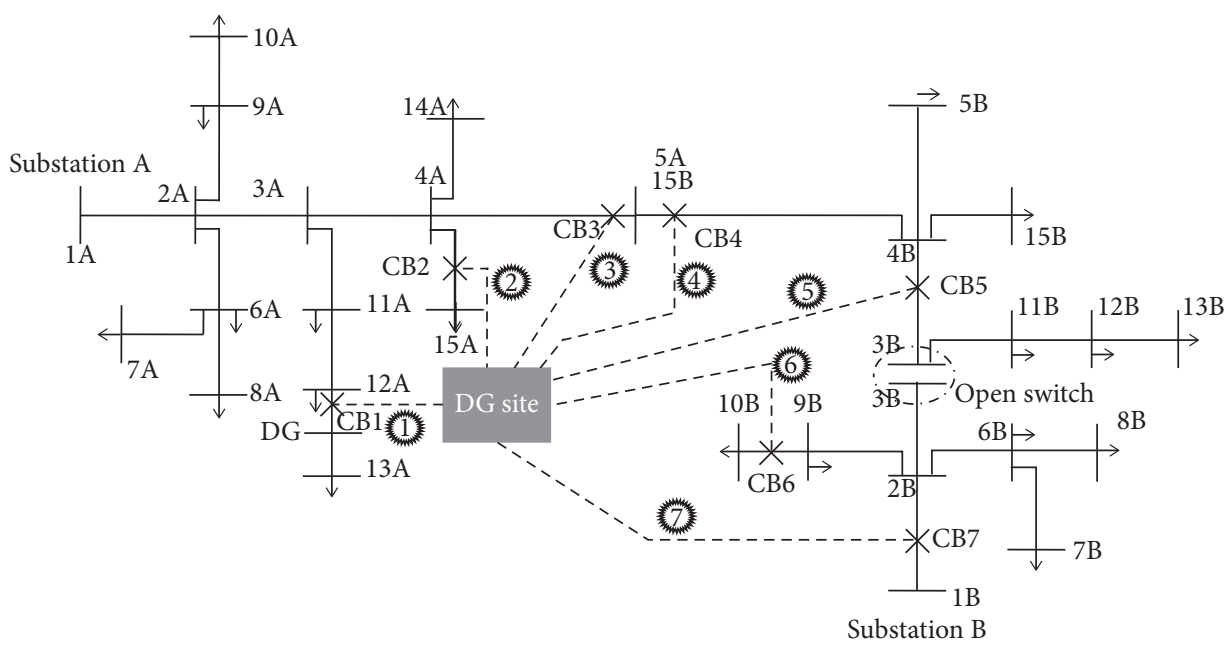

FIgURE 2: Single line diagram of topology no. 2.

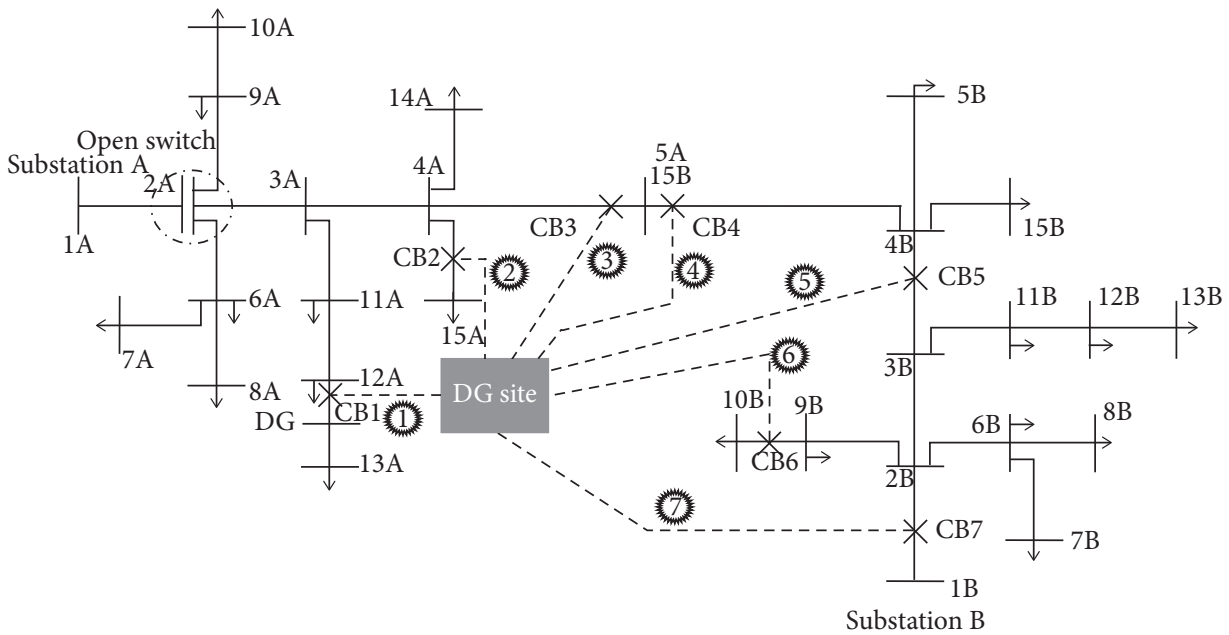

Figure 3: Single line diagram of topology no. 3.

CB2; the impact on the voltage is multiplied by 7 if the network topology changes from structure 1 to structure 3 .

However, the order of the most impacted nodes is still the same; the voltage of the nearest node to the connection point is the most impacted by the variation introduced by the new DG. This observation can also be concluded from the mathematical formulation of the voltage sensitivity calculation.

This powerful observation is used to identify the optimal placement of capacitor units to add (steps 6,7 , and 8 in Section 6) in order to alleviate network voltage constraints and reduce the interconnection cost of renewable distributed generation integration in public medium voltage distribution systems.

\section{Proposed Approach}

The main aim of the proposed approach is to give more options to connect a new DG to a public medium voltage distribution network and offer more chances to get the lowest way to connect a new renewable DG.

As mentioned in Section 2, to connect their DG, the owners will have to address a formal request to the electric utility, who evaluate all the possible connection points with their respect to the operating network constraints; if at least one of the constraints is not satisfied, the connection point is rejected, even if it may present the lower cost to connect the DG to network.

The aim of the proposed approach is to identify the optimal allocation of capacity banks to reduce the impact of the new DG on network voltage constraints. Without the proposed approach, two kinds of solution will be presented to the DG owners: possible connection points, which satisfied all the networks constraints, and no possible connection points, in which at least one of the constraints is not satisfied. With the proposed approach possible, three kinds of solution will be presented: possible connection, which satisfied all the networks constraints, possible connection 
TABLE 1: Voltage sensitivity analysis with respect to the variations of active and reactive power variations.

\begin{tabular}{|c|c|c|c|c|c|c|}
\hline & \multicolumn{2}{|c|}{ Topology no. 1} & \multicolumn{2}{|c|}{ Topology no. 2} & \multicolumn{2}{|c|}{ Topology no. 3} \\
\hline & $\begin{array}{c}\text { Voltage sensitivity } \\
\text { with respect to active } \\
\text { power variation }\end{array}$ & $\begin{array}{l}\text { Voltage sensitivity } \\
\text { with respect to } \\
\text { reactive power } \\
\text { variation }\end{array}$ & $\begin{array}{l}\text { Voltage sensitivity } \\
\text { with respect to } \\
\text { active power } \\
\text { variation }\end{array}$ & $\begin{array}{l}\text { Voltage sensitivity } \\
\text { with respect to } \\
\text { reactive power } \\
\text { variation }\end{array}$ & $\begin{array}{l}\text { Voltage sensitivity } \\
\text { with respect to } \\
\text { active power } \\
\text { variation }\end{array}$ & $\begin{array}{c}\text { Voltage sensitivity } \\
\text { with respect to reactive } \\
\text { power variation }\end{array}$ \\
\hline 2 & 1,53 & 1,778 & 1,53 & 1,778 & 7,165 & 8,749 \\
\hline 4 & 2,567 & 2,849 & 2,567 & 2,849 & 5,941 & 7,321 \\
\hline 3 & 2,567 & 2,849 & 2,567 & 2,849 & 7,165 & 8,749 \\
\hline 11 & 3,717 & 4,134 & 3,717 & 4,134 & 8,315 & 10,034 \\
\hline 12 & 4,991 & 5,656 & 4,991 & 5,656 & 9,589 & 11,556 \\
\hline 13 & 5,628 & 6,417 & 5,628 & 6,417 & 10,226 & 12,317 \\
\hline DG & 5,628 & 6,417 & 5,628 & 6,417 & 10,226 & 12,317 \\
\hline
\end{tabular}

point with additional charge of installing capacitor banks and network reinforcement, which does not satisfy the constraints without additional devices, and not possible connection points, which does not satisfy the network constraints even with additional electrical equipment. The obtained results will be costing to identify the lowest option.

If the lowest option is resulting from the second category, the solution presented by electric utility to the DG owners to connect their generation unit will be presented as the development of underground cables or overhead lines from their DG site to the connection point with additional charge of acquisition, installation, and exploration of capacitor banks. The solution could also be extended for other electrical equipment: energy storage units, D-Statctom, D-FACTS, or Soft Open Points (SOPs).

To regulate the reactive power of the additional devices, several powerful local and decentralized approaches had been developed in [41, 42]. In [41], authors had developed a gradient projection based schemes explicitly accounting for the reactive limit of each bus with the use of only local bus voltage measurements. In [42], three strategies are proposed to keep the voltages within preassigned operating limits, by commanding the reactive power output of the microgenerators connected to the grid; the first two strategies are purely local, each microgenerator updates the amount of reactive power to be injected based only on local measurements of the voltages' magnitude. Instead, the third one is distributed, namely, the microgenerators, to perform the updating steps, requiring some additional information coming from neighboring agents.

\section{Problem Formulation}

Optimal DG access point in a public distribution system is to find best locations of network that gives minimum shortest distance from the DG site to that location, while satisfying certain operating constraints and distribution network actors interests. The operating constraints are voltage profile of the system, current capacity of the feeder and transformers, and the level of the total power losses, energy quality, and protection system limits. The objective function is interconnection cost reduction of a renewable distributed generation integration in public medium voltage distribution systems, which represents the cost required for the construction of overhead lines and underground cables between the generation land use and connection point in the distribution network

$$
F=\min \left(\text { Cost }_{\mathrm{DG}} \text { interconnection }\right),
$$

subject to the following:

(i) Voltage limits: the bus voltage magnitudes are to be kept within acceptable operating limits throughout the optimization process:

$$
V_{\text {Limit-min }} \leq V_{i} \leq V_{\text {Limit-max }}
$$

where $V_{i}$ is The voltage magnitude at node " $i$ ". $V_{\text {Limit-min }}$ and $V_{\text {Limit-max }}$ are the acceptable operating limits of voltage magnitude.

(ii) Thermal limits of lines and transformers:

$$
I_{i j} \leq I_{\text {Limit-max }}
$$

where $I_{i j}$ is the current passing between node " $i$ " and node " $j$ ". $I_{\text {Limit-max }}$ is the acceptable thermal limits of the branch between node " $i$ " and node " $j$."

(iii) Power losses degradation: electric utilities are generally the entity responsible to keep the losses at lower level, to respect their interests; the new DG should not increase the total power losses of the system. The total power losses, before connecting the DG, should not be greater before the DG was connected:

$$
\begin{gathered}
P_{\text {Loss }}^{\text {Before }}-P_{\text {Loss }}^{\text {After }} \geq 0, \\
Q_{\text {Loss }}^{\text {Before }}-Q_{\text {Loss }}^{\text {After }} \geq 0,
\end{gathered}
$$

where $P_{\text {Loss }}^{\text {Before }}$ and $Q_{\text {Loss }}^{\text {Before }}$ are the active and reactive power losses before the introduction of the DG unit. $P_{\mathrm{Loss}}^{\text {After }}$ and $Q_{\mathrm{Loss}}^{\text {After }}$ are the active and reactive power losses after the integration of the DG unit. 
(iv) Total harmonic limits: the total harmonic level at each bus should be less or equal to the maximum allowable harmonic level. Consumers connected at the MV network should not be injured too by connecting the new DG; to preserve a certain quality level at their substations, MV consumers add several devices. Any degradation in energy quality levels signify further charges for MV consumers:

$$
\mathrm{THD}_{i}(\%) \leq \mathrm{THD}_{\max },
$$

where $\mathrm{THD}_{i}(\%)$ is maximum harmonic level at each bus. $\mathrm{THD}_{\max }$ is maximum harmonic operating limit.

(v) Short circuit power constraint:

$$
S_{\mathrm{SC}}^{\prime}<\alpha \cdot S_{\mathrm{SC}}
$$

where $S_{S C}^{\prime}$ and $S_{S C}$ are the short circuit capacity of the network before and after the connection of the DG unit at CBi. $\alpha$ is a factor to apply considering a predetermined safety margin; in [32], the safety margin had been defined as $15 \%$ and $\alpha=0,85$.

(vi) False tripping:

$$
1,2 I_{\mathrm{SC}-\text { adj }}<0,8 I_{P}^{\prime}
$$

where $I_{\mathrm{SC}-\text { adj }}$ is the fault current seen by the relay when of the maximum fault occurs in an adjusted feeder and $I_{P}^{\prime}$ is pickup current of the normal exploration scheme and the backup connections in case of meshed feeders, after the connection of the DG unit.

(vii) Protection blinding:

$$
0,8 I_{S C \text {-min-feeder }}^{\prime}<1,3 I_{p \text {-feeder }}^{\prime}
$$

where $I_{S C \text {-min-feeder }}^{\prime}$ is the minimum short circuit fault current on the MV feeder after the connection of the DG unit and $I_{p \text {-pickup }}^{\prime}$ is pickup current of the normal exploration scheme and the backup connections in case of meshed feeder after the connection of the DG unit.

It is to notice that if the electric utility predicts to reduce the total power losses or a MV consumer needs to improve voltage profile or energy quality level, by changing the connection-point and proposing another access location, a comprise investment could be negotiated between DG owners and the other actors to cover the additional costs of connecting the new DG to the public network.

\section{Algorithm}

The proposed algorithms evaluate all possible connection point under all possible network topology structures; three kinds of solutions will have presented the following: possible connection, which satisfied all the networks constraints, possible connection point with additional charge of installing capacitor banks, which does not satisfy the constraints without additional devices, and not possible connection points, which does not satisfy the network constraints even with additional electrical equipment. The obtained results should be costing separately to identify the lowest option:

(i) Step 1: identify all possible connection-point "CB" to connect the new DG.

(ii) Step 2: arrange the possible connection-point "CB" with their distance to the DG site.

(iii) Step 3: place the new DG at the first connectionpoint

(iv) Step 4: run the load flow analysis of the new network with an additional node on which the new DG is connected, under all possible network topology structures. And also, under different load conditions: morning/afternoon/night, summer/ winter, week/days/weekend.

(v) Step 5: evaluate the voltage constraints for each topology structure, and also, under different load conditions: morning/afternoon/night, summer/ winter, week days/weekend:

If voltage constraints are satisfied go to step 6 . If at least the voltage of one node is not within the permitted range go to step 7 .

(vi) Step 6: evaluate the network electrical constraints, equations (5)-(11), for each topology structure, under different load conditions: morning/afternoon/night, summer/winter, week/days/ weekend:

If all constraints are satisfied the connection point is accepted as possible access point, go to step 3 . If at least one of the constraints are not satisfied, the connection point is rejected, go to step 3 .

(vii) Step 7: place the capacitor bank at the nearest nodes to the DG connection-point.

(viii) Step 8: increase the capacitor banks size.

(ix) Step 9: run the load flow analysis of the new network with an additional node on which the new DG is connected, under all possible network topology structures. And also, under different load conditions: morning/afternoon/night, summer/ winter, week/days/weekend.

(x) Step 10: evaluate the voltage constraints for each topology structure, and also, under different load conditions: morning/afternoon/night, summer/ winter, weekdays/weekend.

If voltage constraints are satisfied, go to step 9. If at least the voltage of one node is not within the permitted range, go to step 7.

(xi) Step 11: evaluate the network electrical constraints, equations (5)-(11), under all possible network topology structures, and also under different load 


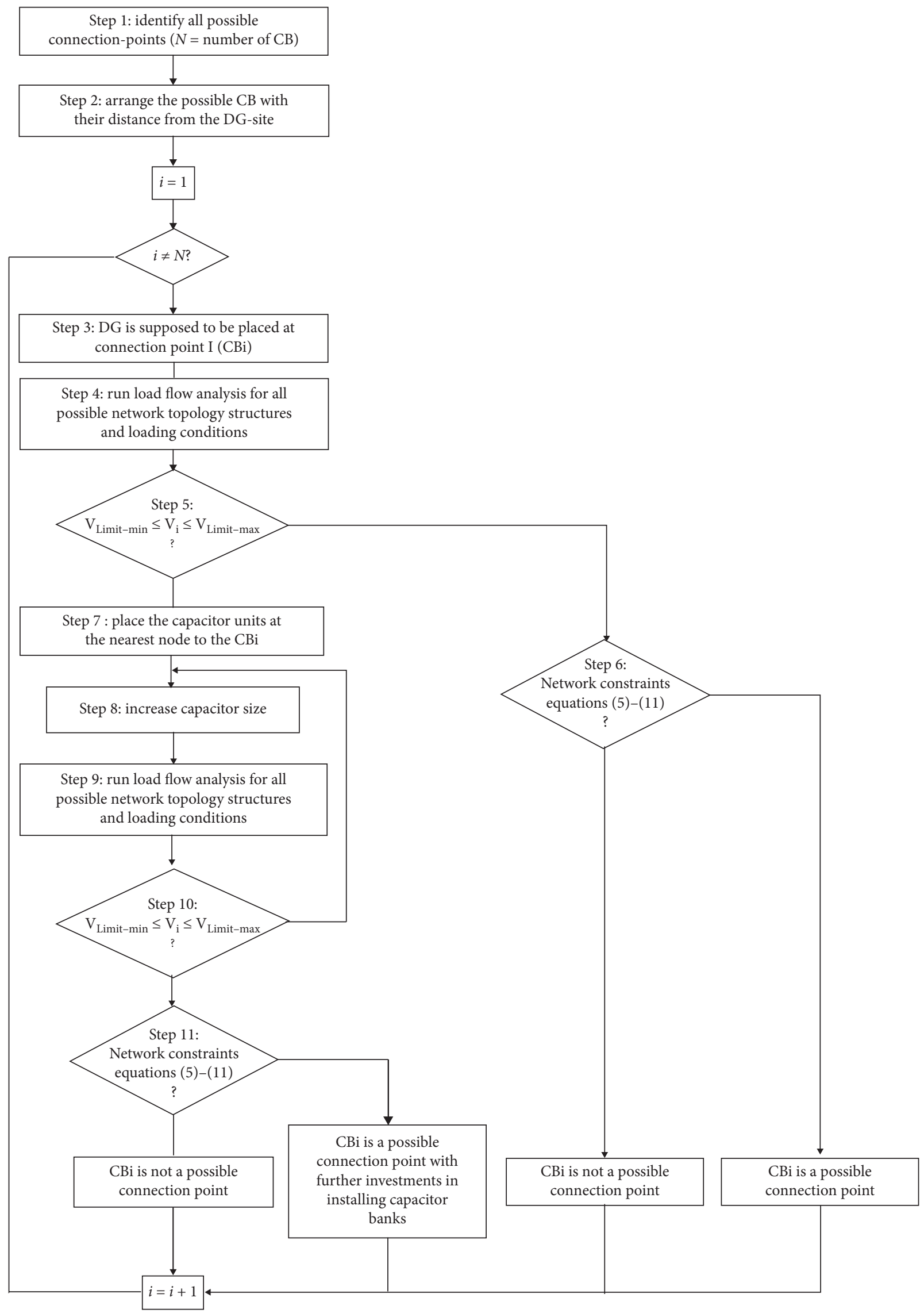

Figure 4: Flow chart of the proposed approach. 
TABLE 2: Load data and line data of the two meshed feeders A and B (feeder A and feeder B are identical).

\begin{tabular}{|c|c|c|c|c|c|c|c|}
\hline Sending node (A) & Receiving node (A) & Sending node (B) & Receiving node (A) & $R \mathrm{Ohm}$ & $X$ Ohm & $\mathrm{kVA}$ & $\mathrm{kVAr}$ \\
\hline 1 & 2 & 1 & 2 & 1.353 & 1.323 & 44.1 & 44.99 \\
\hline 2 & 3 & 2 & 3 & 1.170 & 1.144 & 20.1 & 11.44 \\
\hline 3 & 4 & 3 & 4 & 0.841 & 0.822 & 40 & 142.8 \\
\hline 4 & 5 & 4 & 5 & 1.523 & 1.027 & 44.1 & 44.99 \\
\hline 2 & 9 & 2 & 9 & 2.013 & 1.327 & 10 & 4.82 \\
\hline 9 & 10 & 9 & 10 & 1.686 & 1.137 & 10 & 4.82 \\
\hline 2 & 6 & 2 & 6 & 2.557 & 1.724 & 20 & 21.41 \\
\hline 6 & 7 & 6 & 7 & 1.088 & 0.734 & 20 & 21.44 \\
\hline 6 & 8 & 6 & 8 & 1.251 & 0.844 & 14.1 & 9 \\
\hline 3 & 11 & 3 & 11 & 1.795 & 1.211 & 30 & 10.82 \\
\hline 11 & 12 & 11 & 12 & 2.448 & 1.651 & 20 & 11.41 \\
\hline 12 & 13 & 12 & 13 & 2.013 & 1.357 & 44.1 & 44.99 \\
\hline 4 & 14 & 4 & 14 & 2.230 & 1.504 & 70 & 71.41 \\
\hline 4 & 15 & 4 & 15 & 1.970 & 0.807 & 40 & 42.82 \\
\hline
\end{tabular}

conditions: morning/afternoon/night, summer/ winter, week/days/weekend.

If all constraints are satisfied the connection point is accepted as possible access point with an additional investment on acquisition and installation of further capacity bank, go to step 3 .

If at least one of the constraints are not satisfied, the connection point is rejected, go to step 3 .

The results will be presented as possible scenarios to connect the new DG. Without the proposed approach, the connection point which had not satisfied the electrical network constrains is directly rejected, but with the proposed method, some of those connection points could be accepted as possible access point of the new renewable DG but with further investment of acquisition and installation of capacity banks.

The cost of connecting DGs to an existing MV network will be reduced by this approach by choosing the shortest electrical connection and acquisition capacity banks, instead of choosing a largest electrical connection.

The cost of capacity bank is very low compared to the cost of underground MV cable lying or the development of MV overhead lines. Also, the cost of an electrical connection does not only depend on the length of cables, the nature of the geographical terrain, and the presence of constraints influence the total cost of the electrical connection.

The proposed method offers more options to connect a new DG to an existing MV network, all the solution resulting from the approach should be evaluated to calculate the total cost of connecting the new DG.

The evaluation of all network topology structures needs high computational effort, which impacts the time application; however, this limitation is not necessarily critical in DG placement applications.

The flow chart of the possible algorithm is given in Figure 4.

\section{Simulation Results}

The proposed analytical approach had been applied to the electrical system, presented in Figure 1, composed of two meshed feeders of 15 buses, driven from two separate HV/
TABLE 3: Distance between possible connection point and DG site.

\begin{tabular}{lcccccc}
\hline CB1 & CB2 & CB3 & CB4 & CB5 & CB6 & CB7 \\
\hline $1270 \mathrm{~m}$ & $2404 \mathrm{~m}$ & $2446 \mathrm{~m}$ & $2630 \mathrm{~m}$ & $2815 \mathrm{~m}$ & $3007 \mathrm{~m}$ & $3085 \mathrm{~m}$
\end{tabular}

MV substations A and B and a new DG of $1500 \mathrm{~kW}$ to connect. The line data and load data of the electrical system are given in Table 2, and distance from the DG unit seven possible connection points is given in Table 3 .

Firstly, the seven possible connection points CBi are tested considering all possible topology structure of the two feeders, obtained by changing the sectionalize switches and tie switches of all system nodes.

The results of the maximum and the minimum voltage magnitude for the three different topology structures are provided in Table 4.

The results obtained in Table 4 show that CB1 is not a possible access point to connect the generation unit. Connecting the new DG at CB1 may create an overvoltage when structure 3 is the topology structure of the electric system.

By applying the proposed approach, the optimal allocation of reactive power devices obtained are two capacitors banks of $100 \mathrm{kVAr}$ at nodes " $12 \mathrm{~A}$ " and " $13 \mathrm{~A}$." The system performance after the connection of the new DG at CB1 and the installation of the two capacitor banks at the optimal locations is given in Table 5 .

Without the application of the proposed method, the nearest possible access point for the new DG is CB2; however, with the introducing of the proposed approach, DG owners will be able to connect their DG unit at CB1, which presents half the length of cables needed to lay to reach the network, but with additional charges of installing and exploring two capacitors banks of $100 \mathrm{kVAr}$.

An estimation of connecting the new DG at CB1 with 2 additional capacitor banks of $100 \mathrm{kVAr}$ is 183060.00 Moroccan Dirham, including taxes (19262.49 US dollar), needed to develop and install 10 electric MV cement poles of 12 meter/ $300 \mathrm{daN}, 4$ electric poles of 12 meter/500 daN, 14 electrical cross arms, 54 composite insulators, 1 disconnect switch, 3810 meters of conductor's aluminum alloy (Almelec) with a section $34.4 \mathrm{~mm}^{2}$, and 2 capacitors banks of $100 \mathrm{kVAr}$. 
TABLE 4: Evaluation of the seven connection points with respect to the voltage profile under three different topology structures.

\begin{tabular}{llclllllll}
\hline & & Before DG & CB1 & CB2 & CB3 & CB4 & CB5 & CB6 & CB7 \\
\hline \multirow{2}{*}{ Structure 1 } & $V_{\max }$ & 0.98 & 1.06 & 1.05 & 1.04 & 1.05 & 1.03 & 1.03 & 1.00 \\
& $V_{\min }$ & 0.97 & 1.00 & 1.01 & 1.00 & 1.01 & 1.01 & 0.99 & 0.99 \\
\hline \multirow{2}{*}{ Structure 2 } & $V_{\max }$ & 0.97 & 1.05 & 1.03 & 1.01 & 1.03 & 1.04 & 1.08 & 1.06 \\
& $V_{\min }$ & 0.92 & 0.96 & 0.97 & 0.99 & 0.99 & 0.99 & 0.99 & 0.99 \\
\hline \multirow{2}{*}{ Structure 3 } & $V_{\max }$ & 0.97 & $\mathbf{1 . 1 0}$ & 1.06 & 1.01 & 1.00 & 1.00 & 1.02 & 0.99 \\
& $V_{\min }$ & 0.92 & 0.99 & 0.99 & 0.99 & 0.98 & 0.96 & 0.94 & 0.94 \\
\hline
\end{tabular}

TABLE 5: Voltage profile before the connection of the new DG, after the connection at CB1, with and without the proposed method.

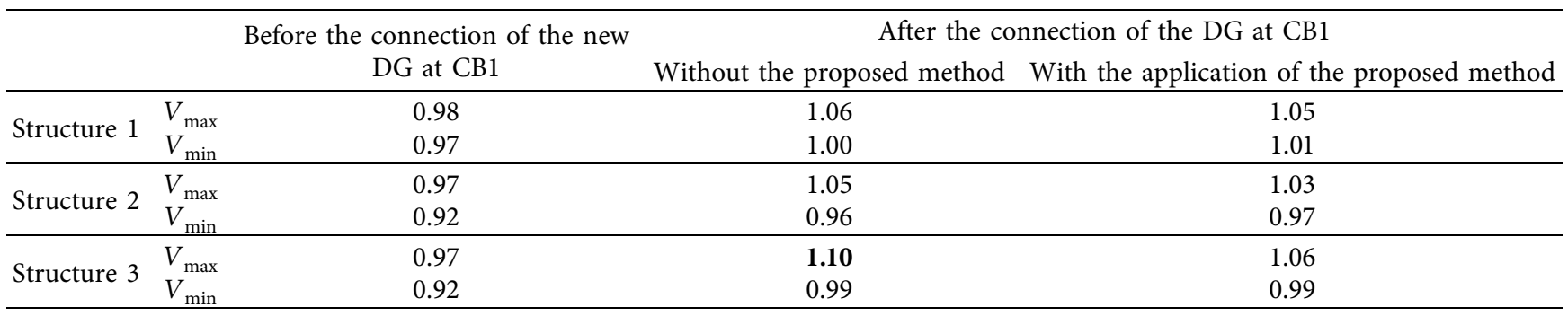

Without the proposed solution, the new DG will be connected at CB2, with a cost of 283545.00 Moroccan Dirham, including taxes (29836.02 US dollar) to develop an overhead line of $20 \mathrm{MV}$ electric cement poles of 12 meter/ 300 daN, 7 electric poles of 12 meter/500 daN, 27 electrical cross arms, 102 composite insulators, 1 disconnect switch, and 7212 meters of conductor's aluminum alloy (Almelec) with a section $34.4 \mathrm{~mm}^{2}$, which clearly confirm the ability of the proposed approach to reduce the cost of connecting a renewable generation unit to a medium voltage distribution network.

\section{Conclusions}

This paper presented an analytical approach for optimally allocating renewable distribution generations and capacitor banks, with a main aim of minimizing the cost of connecting a new renewable DG unit to public medium voltage distribution systems, considering practical constraints of network topology reconfigurations and the presence of several actors with different interests in a competitive electrical market.

Simulation results obtained from a two meshed feeders showed that the proposed method is able to minimize the total length of electrical cables and lines needed to connect a new generation unit to a public distribution network while satisfying actors interests and network constraints.

The approach presented in this paper was developed based on an analytical approach; several heuristics and numerical methods are more robust and more efficient and could be used for future improvement. Further constraints could be adopted as time varying of load demand and renewable generation and also the consideration of load growth, if multiple years are considered and an optimal DG placement along a planning horizon is searched. Further actuators devices could also be added for further improvement as energy storage units, D-Statctom, D-FACTS, or Soft Open Points (SOPs).

\section{Data Availability}

The code MATLAB developed and used to perform the proposed method is available from the corresponding author upon request.

\section{Conflicts of Interest}

The authors declare that there are no conflicts of interest regarding the publication of this paper.

\section{References}

[1] P. Dehghanian, S. H. Hosseini, M. Moeini-Aghtaie, and A. Arabali, "Optimal siting of DG units in power systems from a probabilistic multi-objective optimization perspective," International Journal of Electrical Power \& Energy Systems, vol. 51, pp. 14-26, 2013.

[2] E. E. Sfikas, Y. A. Katsigiannis, and P. S. Georgilakis, "Simultaneous capacity optimization of distributed generation and storage in medium voltage microgrids," International Journal of Electrical Power \& Energy Systems, vol. 67, pp. 101-113, 2015.

[3] A. Keane and M. O'Malley, "Optimal allocation of embedded generation on distribution networks," IEEE Transactions on Power Systems, vol. 20, no. 3, pp. 1640-1646, 2005.

[4] S. Kansal, V. Kumar, and B. Tyagi, "Optimal placement of different type of DG sources in distribution networks," International Journal of Electrical Power \& Energy Systems, vol. 53, pp. 752-760, 2013.

[5] R. S. Rao, K. Ravindra, K. Satish, and S. V. L. Narasimham, "Power loss minimization in distribution system using network reconfiguration in the presence of distributed 
generation," IEEE Transactions on Power Systems, vol. 28, no. 1, pp. 317-325, 2013.

[6] K. M. Muttaqi, A. D. T. Le, M. Negnevitsky, and G. Ledwich, "An algebraic approach for determination of DG parameters to support voltage profiles in radial distribution networks," IEEE Transactions on Smart Grid, vol. 5, no. 3, pp. 1351-1360, 2014.

[7] R. S. Al-Abri, E. F. El-Saadany, and Y. M. Atwa, "Optimal placement and sizing method to improve the voltage stability margin in a distribution system using distributed generation," IEEE Transactions on Power Systems, vol. 28, no. 1, pp. 326334, 2014.

[8] W. EI-Khattam, K. Bhattacharya, Y. G. Hagazy, and M. M. A. Salama, "Optimal investment planning for distributed generation in a competitive electricity market," IEEE Transactions on Power Systems, vol. 19, no. 3, pp. 1674-1684, 2004.

[9] M. F. Shaaban, Y. M. Atwa, and E. F. El-Saadany, "DG allocation for benefit maximization in distribution networks," IEEE Transactions on Power Systems, vol. 28, no. 2, pp. 639-649, 2013.

[10] S. Abdi and K. Afshar, "Application of IPSO-Monte Carlo for optimal distributed generation allocation and sizing," International Journal of Electrical Power \& Energy Systems, vol. 44, no. 1, pp. 786-797, 2013.

[11] D. Gautam and N. Mithulananthan, "Optimal DG placement in deregulated electricity market," Electric Power Systems Research, vol. 77, no. 12, pp. 1627-1636, 2007.

[12] J.-H. Teng, Y.-H. Liu, C.-Y. Chen, and C.-F. Chen, "Valuebased distributed generator placements for service quality improvements," International Journal of Electrical Power \& Energy Systems, vol. 29, no. 3, pp. 268-274, 2007.

[13] G. N. Koutroumpezis and A. S. Safigianni, "Optimum allocation of the maximum possible distributed generation penetration in a distribution network," Electric Power Systems Research, vol. 80, no. 12, pp. 1421-1427, 2010.

[14] S. N. G. Naik, D. K. Khatod, and M. P. Sharma, "Analytical approach for optimal sitting and sizing of distributed generation in radial distribution networks," IET Generation, Transmission \& Distribution, vol. 9, no. 3, pp. 209-220, 2015.

[15] M. F. AlHajri, M. R. AlRashidi, and M. E. El-Hawary, "Improved sequential quadratic programming approach for optimal distribution generation deployments via stability and sensitivity analyses," Electric Power Components and Systems, vol. 38, no. 14, pp. 1595-1614, 2010.

[16] A. C. Rueda-Medina, J. F. Franco, M. J. Rider, A. PadilhaFeltrin, and R. Romero, "A mixed-integer linear programming approach for optimal type, size and allocation of distributed generation in radial distribution systems," Electric Power Systems Research, vol. 97, pp. 133-143, 2013.

[17] Y. M. Atwa, E. F. El-Saadany, M. M. A. Salama, and R. Seethapathy, "Optimal renewable resources mix for distribution system energy loss minimization," IEEE Transactions on Power Systems, vol. 25, no. 1, pp. 360-370, 2010.

[18] N. Kanwar, N. Gupta, K. R. Niazi, A. Swarnkar, and R. C. Bansal, "Simultaneous allocation of distributed energy resource using improved particle swarm optimization," $A p$ plied Energy, vol. 185, pp. 1684-1693, 2017.

[19] Z. Moravej and A. Akhlaghi, "A novel approach based on cuckoo search for DG allocation in distribution network," International Journal of Electrical Power \& Energy Systems, vol. 44, no. 1, pp. 672-679, 2013.

[20] S. K. Goswami and R. K. Singh, "Optimum sitting and sizing of distributed generations in radial and networked systems,"
Electric Power Components and Systems, vol. 37, no. 2, pp. 127-145, 2009.

[21] S. H. Lee and J. W. Park, "Selection of optimal location and size of multiple distributed generations by using kalman filter algorithm," IEEE Transactions on Power Systems, vol. 24, no. 3, pp. 1393-1400, 2009.

[22] L. D. Arya, A. Koshti, and S. C. Choube, "Distributed generation planning using differential evolution accounting voltage stability consideration," International Journal of Electrical Power \& Energy Systems, vol. 42, no. 1, pp. 196-207, 2012.

[23] S. Saha and V. Mukherjee, "Optimal placement and sizing of DGs in RDS using chaos embedded SOS algorithm," IET Generation, Transmission \& Distribution, vol. 10, no. 14, pp. 3671-3680, 2016.

[24] K. Nara, Y. Hayashi, K. Ikeda, and T. Ashizawa, "Application of tabu search to optimal placement of distributed generators," in Proceedings of the 2001 IEEE Power Engineering Society Winter Meeting. Conference, pp. 918-923, Columbus, $\mathrm{OH}$, USA, Febraury 2001.

[25] K. Nekooei, M. M. Farsangi, H. Nezamabadi-Pour, and K. Y. Lee, "An improved multi-objective harmony search for optimal placement of DGs in distribution systems," IEEE Transactions on Smart Grid, vol. 4, no. 1, pp. 557-567, 2013.

[26] M. H. Sulaiman, M. W. Mustafa, A. Azmi, O. Aliman, and S. R. Abdul Rahim, "Optimal allocation and sizing of distributed generation in distribution system via firefly algorithm," in Proceedings of the 2012 IEEE International Power Engineering and Optimization Conference, pp. 84-89, Melaka, Malaysia, June 2012.

[27] A. I. Aly, Y. G. Hegazy, and M. A. Alsharkawy, "A simulated annealing algorithm for multi-objective distributed generation planning," in Proceedings of the Power and Energy Society General Meeting, pp. 1-7, IEEE, Providence, RI, USA, July 2010.

[28] U. Sultana, A. B. Khairuddin, A. S. Mokhtar, N. Zareen, and B. Sultana, "Grey wolf optimizer based placement and sizing of multiple distributed generation in the distribution system," Energy, vol. 111, pp. 525-536, 2016.

[29] N. D. Mahmoudabadi, B. F. Moghadam, and H. kazemipoor, "Optimal allocation and sizing of distributed generation in distribution network using modified shuffled leaping algorithm," Saussurea, vol. 5, no. 6, pp. 75-86, 2015.

[30] M. Esmaeili, M. Sedighizadeh, and M. Esmaili, "Multi-objective optimal reconfiguration and DG (distributed generation) power allocation in distribution networks using big bang-big crunch algorithm considering load uncertainty," Energy, vol. 103, pp. 86-99, 2016.

[31] S. Devi and M. Geethanjali, "Application of modified bacterial foraging optimization algorithm for optimal placement and sizing of distributed generation," Expert Systems with Applications, vol. 41, no. 6, pp. 2772-2781, 2014.

[32] L. Wang and C. Singh, "Reliability-constrained optimum placement of reclosers and distributed generators in distribution networks using an ant colony system algorithm," IEEE Transactions on Systems, Man, and Cybernetics, Part C (Applications and Reviews), vol. 38, no. 6, pp. 757-764, 2008.

[33] M. Gandomkar, M. Vakilian, and M. Ehsan, "Optimal distributed generation allocation in distribution network using Hereford Ranch algorithm," in Proceedings of the 2005 International Conference on Electrical Machines and Systems, vol. 2, pp. 916-918, Nanjing, China, September 2005.

[34] F. S. Abu-Mouti and M. E. El-Hawary, "Optimal distributed generation allocation and sizing in distribution systems via 
artificial bee colony algorithm," IEEE Transactions on Power Delivery, vol. 26, no. 4, pp. 2090-2101, 2011.

[35] T. Niknam, S. I. Taheri, J. Aghaei, S. Tabatabaei, and M. Nayeripour, "A modified honey bee mating optimization algorithm for multiobjective placement of renewable energy resources," Applied Energy, vol. 88, no. 12, pp. 4817-4830, 2011.

[36] M. H. Moradi and M. Abedini, "A combination of genetic algorithm and particle swarm optimization for optimal DG location and sizing in distribution systems," International Journal of Electrical Power \& Energy Systems, vol. 34, no. 1, pp. 66-74, 2012.

[37] M. Gandomkar, M. Vakilian, and M. Ehsan, "A genetic-based tabu search algorithm for optimal DG allocation in distribution networks," Electric Power Components and Systems, vol. 33, no. 12, pp. 1351-1362, 2005.

[38] M. Gandomkar, M. Vakilian, and M. Ehsan, "A combination of genetic algorithm and simulated annealing for optimal DG allocation in distributed networks," in Proceedings of IEEE Electrical and Computer Engineering Canadian Conference, pp. 645-648, Saskatoon, Sask., Canada, May 2005.

[39] S. Montoya-Bueno, J. I. Muñoz, and J. Contreras, "A stochastic investment model for renewable generation in distribution systems," IEEE Transactions on Sustainable Energy, vol. 6, no. 4, pp. 1466-1474, 2015.

[40] J.-F. Toubeau, F. Vallée, Z. De Grève, and J. Lobry, "A new approach based on the experimental design method for the improvement of the operational efficiency in medium voltage distribution networks," International Journal of Electrical Power \& Energy Systems, vol. 66, pp. 116-124, 2015.

[41] Z. Hao and J. L. Hao, "Fast local voltage control under limited reactive power: optimality and stability analysis," IEEE Transactions on Power Systems, vol. 31, pp. 360-370, 2016.

[42] C. Guido and C. Ruggero, "Local and distributed voltage control algorithms in distribution networks," IEEE Transactions on Power Systems, vol. 32, no. 2, pp. 560-570, 2018. 